\title{
Risk analysis in digital twin creation of machine building production
}

\author{
Alexander Feofanov ${ }^{1}$ and Nikolay Baranov ${ }^{2, *}$ \\ ${ }^{1}$ Moscow State University of Technology “STANKIN”, 127055 Moscow, Russia \\ ${ }^{2}$ Saint-Petersburg State University of Civil Aviation, 196210Saint-Petersburg, Russia
}

\begin{abstract}
Digital twin is one of instruments of Industry 4.0 in order to effectively manage the enterprise and its activities. However, the digital twin of production application is exposed to various risks. Careful identification of these risks speeds up digital twin creation and implementation process.
\end{abstract}

The current stage of an enterprise development in any sphere of the economy is characterized by a continuous complication of technological processes, an increase in the level of competition, and an ever-growing volume of data that must be interpreted, processed, and analyzed. At the moment information is one of the key enterprise resources and the efficiency of information processing determines the effective work of the entire enterprise. Given the advent of the digitalization era, it seems relevant to use various instruments of Industry 4.0 in order to effectively manage the enterprise and its activities. One such instrument is a digital twin of production [1, 2].

A digital twin is a virtual copy of the physical world, a virtual model of a real object or situation, which at the micro and macro level using a set of mathematical models describes the state of the object and all its elements. This can be achieved due to the fact that the mathematical model of production or an object, presented in a digital environment, is connected with a database of parameters of this object. When one of the parameters is changed, the remaining parameters and objects associated with it are automatically changed. The digital twin is not limited to collecting data obtained at the stage of product development and manufacturing - it continues to collect and analyze data during the entire life cycle of a real object. This allows you to create valuable feedback channels. [2, 3].

There is a unique opportunity to simulate the enterprise activities without real changes in technological processes, supply chains, the location of means of production. The virtual model helps with a high adequacy to evaluate how certain changes will affect the operation of the enterprise, excluding long, expensive and risky experiments in real production. Moreover, the digital model is constantly updated, thus ensuring maximum compliance with the current operating mode of the real situation in the workplace. Using a digital twin, you can work out many options for organizing technological, production, and logistics processes.Based on the virtual results obtained, it becomes possible to understand which decisions need to be taken and which should be discarded without the risk of failure of

\footnotetext{
*Corresponding author: baranov@post.com
} 
established functioning processes that can lead to interruptions in production process, and, as a result, to financial losses. Using the digital twin methods, it is possible to reduce by an order of magnitude the number of field tests, the number of attempts to develop technological processes, all those actions associated with the manufacture of the real material part and its tests, the cost of which is significantly higher than the cost of mathematical modeling. In other words, the digital twin of production increases the predictability and transparency of enterprise management.

The theory underlying the concept of the digital twinwas developed about 15 to 20 years ago. However, only now the hardware has reached the level at which we can talk about the full-scale implementation of the digital twin strategy in real production [1].

Each structure is determined by a set of elements and the type of their interaction between themselves and with the external environment in different conditions. The elements of architecture, being interconnected, form the unity of the management system, distinguish it from other organizational systems and provide a solution to the tasks assigned to it at the architectural level [4].

Thus, the key problem in solving the task of creating and implementing a digital twin of production is its comprehensive, balanced development, interconnected implementation of the whole range of activities aimed at creating promising examples of systems and complexes of automation and telecommunications, taking into account the scientific and technical groundwork for innovation, the possibilities of industrial complex and the country's economy as a whole, promising areas and world experience in the development of production in general, manifestations of external and internal destabilizing factors, including the emergence of new problems and the presence of different kinds of resource constraints.

This problem can only be solved by dynamically adapting the architectures of the digital twin to the prevailing conditions due to mutual compensation or complementing the characteristics of some structural elements with the characteristics of other elements or the relationships between them. At the same time, to solve this problem, it is necessary to develop a method for dynamically adapting the architecture of a digital twin creation, taking into account the forecast for the development of a complex of factors of various properties that determine the effectiveness of its functioning, as well as the risks of both the Developer and the Customer. Therefore, the use of qualimetric looks very promising methods to solve this problem.

One of the approaches to designing an effective digital twin architecture is to ensure its balance, taking into account resource constraints and expected operating conditions. When implementing this approach, it is necessary to take into account that one of the features of the digital twin is the presence in its composition of components related to different vendors and generations.

Aggregation of these factors into a system, their classification will allow you to create a problem situation and get to the problems associated with overcoming the main obstacles to the implementation of new information technologies in production.

By analogy with the reasons for the failure to introduce ERP-systems in industrial enterprises, we formulate the following main problems associated with the process of creating and implementing a digital twin of engineering production[5]:

1. Features of the organization and management of the enterprises themselves. Enterprises come in various sizes, everywhere there is a style and management nuances that must be taken into account when initiating projects. A critical factor is also the requirement to form a high-quality internal team on the customer's side.

2. The level of competence of companies involved in the creation and implementation of digital twin. 
3. The complexity and all-inclusiveness of a digital twin. Such systems are an extremely complex product that affects all significant processes of the enterprise - dozens of departments, hundreds of active users of the system.

4. Industry specifics of enterprises.

5. High cost of the digital twin creation and implemetation.

6. The absence of regulatory acts on the application and use of digital twin strategies.

It is necessary to take into account the fact that the risks in creating and implementing digital twins of complex production are much greater and they must be identified, taken into account and managed during the project.

The psychological factor of the managerial link should also be added: a lack of understanding of the need for automation and, as a result, opposition of the managerial apparatus, starting with the director and above, to innovations.

We list the main some of the risks associated with this factor[6]:

1. Conflict of interests of customer's top managers, in which it is impossible to agree on the goals of the project.

2. Loss of interest in the project by the project owner.

3. Lack of competent persons for separate business processes.

4. Lack of human resources allocated for the development of the system, unmotivated users, without which the successful implementation of a digital twin is almost impossible.

5. Underestimation of the scope of work on the input and reconciliation of data on the creation of regulatory and reference information, which is especially important for machine building.

6. Insufficient number or dismissal of participants in the task force, which is a very serious risk that directly affects the success of the project.

7. Hidden sabotage of employees (managers) by the customer.

8. The desire of the customer to save at any cost without changing the requirements for the result.

Of course, at the moment the digital twin of production application is encountering certain difficulties. However, with the development of scientific and technological progress, the digitalization of the economy, the modern organization is faced with the tasks of introducing new technically sophisticated equipment in production, increasing the complexity of technological processes in the enterprise and increasing the number and nomenclature of manufactured products, the need to increase the competitiveness of the enterprise and quickly adapt to changing market conditions . To obtain an effective solution to such problems only with the help of human efforts to manage the enterprise is not possible. In this regard, the introduction of various automated production management systems, including the digital twin strategy, is relevant and serves the purpose of developing the enterprise and increasing its competitiveness.

\section{References}

1. K.S.Ponomarev, A.N. Feofanov, T.G.Grishina, Proceedings of All-Russian theoretical and practical conference "Digital economy: equipment, management, human capital", Vologda, 2018, pp. 73-76. (in Russian).

2. K.S.Ponomarev, A.N. Feofanov, Proceedings of International theoretical and practical conference "Topical trends and development prospects of science, engineering, techniques, Belgorod, 2018, pp. 141-144. (in Russian).

3. Z.Feuer,Z. Weissman, CAD/CAM/CAE Observer, v. 5 (113), pp. 50-53 (2017). (in Russian). 
4. A.N. Feofanov, N.E. Baranov, Modern science: actual problems of theory and practice, Natural and Technical Sciences series, v. 3, pp. 116-119 (2019). (in Russian).

5. A.N. Feofanov, N.E. Baranov, Proceedings of International theoretical and practical conference "Topical trends and development prospects of science, engineering, techniques, Belgorod, 2018, pp. 153-159. (in Russian).

6. N.E. Baranov, A.N. Feofanov, TekhnologiyaMashinostroeniya, v. 2 (200), pp. 65-71. (2019). (in Russian). 